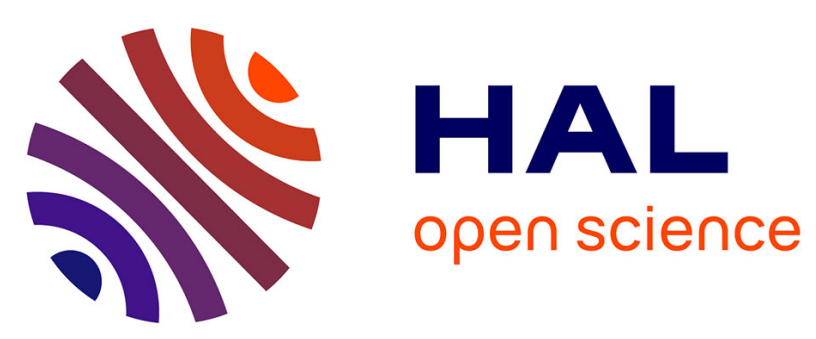

\title{
Optimization of structural dynamic behaviour based on effective modal parameters
}

Sébastien Besset, Louis Jézéquel

\section{To cite this version:}

Sébastien Besset, Louis Jézéquel. Optimization of structural dynamic behaviour based on effective modal parameters. International Journal for Numerical Methods in Engineering, 2007, 70 (5), pp.523542. 10.1002/nme.1890 . hal-00625128

\section{HAL Id: hal-00625128 \\ https://hal.science/hal-00625128}

Submitted on 26 Sep 2011

HAL is a multi-disciplinary open access archive for the deposit and dissemination of scientific research documents, whether they are published or not. The documents may come from teaching and research institutions in France or abroad, or from public or private research centers.
L'archive ouverte pluridisciplinaire HAL, est destinée au dépôt et à la diffusion de documents scientifiques de niveau recherche, publiés ou non, émanant des établissements d'enseignement et de recherche français ou étrangers, des laboratoires publics ou privés. 


\title{
Optimization of Structural Dynamic Behaviour Based on Effective Modal Parameters
}

\author{
S. Besset, L. Jézéquel \\ LTDS, Équipe D2S, UMR CNRS 5513 \\ École Centrale de Lyon \\ 36 av. Guy de Collongue \\ 69134 Ecully cedex, France \\ International Journal for Numerical Methods in Engineering, 70(5), 523-542, 2007
}

\begin{abstract}
Optimization of complex structures often leads to high calculation costs. Indeed, the structure has to be frequently reanalysed in order to update the optimization criteriums. We propose an optimization method based on effective modal parameters. These parameters are close to the modal matrices used for the modal analysis of a structure. Thus, once the structure has been analysed, it becomes very easy to calculate optimization criteria. First, we will explain the modal analysis that we will use in this paper. A modal model will be used to analyse the hollow parts of the structure. The modal analysis of the whole structure will be performed using substructuring and "double modal synthesis" proposed by Jezequel. Secondly, we will explainthe how to obtain effective modal parameters and their use for optimization. Finally, we will show the efficiency of these parameters through the optimization of a complex structure, using two types of optimization methods.
\end{abstract}

keywords: Modal analysis; Optimization; Modal criteria

\section{Introduction}

Using matrices resulting from the modal analysis of a structure in order to optimize the dynamic behaviour of this structure has multiple advantages. Once the structure has been analyzed, optimization criteria become very easy to compute. Secondly, it is possible to link the optimization of the structure and the modal matrices resulting from the modal analysis associated with the resonance phenomena. Thus, it is possible to identify the causes of the problems to solve. The criteria we will use in this paper have been developed by P. Lemerle [1], using the Craig \& Bampton method for structure analysis. The doctoral dissertation of P. Lemerle is derivated in the present paper. Similar optimization methods have already been used by Suweca [2] in the case of structural designs with damping constraint limitations. 
To optimize the structure, we will modify properties of the hollow parts of the structure, but other properties could be changed. The example we propose is a complex structure, including plates and stiffeners, that we will optimize through two types of methods. First, a global method, using a genetic algorithm. Secondly, a local optimization will be processed, using a method based on the gradient method and using Kühn \& Tucker conditions.

\section{Effective modal parameters}

In order to compute the effective modal parameters, we will first explain the modal analysis of the structure used in this study.

\subsection{Modal analysis of a structure}

The structure used in this study is a complex structure including hollow parts and plates. It is made of formed steel constituting its skeleton, as shown in figure 1 Plates are attached to this skeleton. The geometry of the structure is similar to the geometry of a car, in order to demonstrate that the methods we propose can be used in an industrial context.

\section{[Figure 1 about here.]}

Hollow parts of the structure - which could be stiffeners for example - are analyzed using a model we proposed in [3]. These elements constitute the skeleton of the structure. This modelization leads to modal matrices that can be assembled like finite elements matrices. The main characteristic of this method is that it produces matrices including only generalized degrees of freedom. There may remain nodal degrees of freedom in order to assemble the hollow parts with other structures, but boundaries between the elements constituting the hollow part only comprise generalized degrees of freedom. It is possible to assemble these elements because of the choice of modes used for the modal analysis of the elements. We now summarize the method used in [3]. Figures 2 and 3 show the geometry of the hollow parts we consider. The hollow part shown in figure 2 is split into several elements shown in figure 3

[Figure 2 about here.]

[Figure 3 about here.]

When the modal analysis of one element is complete, two nodal degrees of freedom will remain, in order to couple the hollow part with another structure. The behavior of an element can be described with the equation of motion:

$$
\left(-\omega^{2}\left[\begin{array}{ll}
\mathbf{M}_{L L} & \mathbf{M}_{L R} \\
\mathbf{M}_{R L} & \mathbf{M}_{R R}
\end{array}\right]+\left[\begin{array}{ll}
\mathbf{K}_{L L} & \mathbf{K}_{L R} \\
\mathbf{K}_{R L} & \mathbf{K}_{R R}
\end{array}\right]\right)\left\{\begin{array}{l}
\mathbf{u}_{L} \\
\mathbf{u}_{R}
\end{array}\right\}=\left\{\begin{array}{l}
\mathbf{f}_{L} \\
\mathbf{f}_{R}
\end{array}\right\}
$$

where mass and stiffness matrices $\mathbf{M}$ and $\mathbf{K}$ are split into left and right degrees of freedom $L$ and $R$. Vectors $\mathbf{u}$ and $\mathbf{f}$ are split the same way. 
$\mathbf{u}_{L}$ and $\mathbf{u}_{R}$ are then split into degrees of freedom that will be expressed as a function of generalized degrees of freedom $\mathbf{u}_{L}^{g}$ and $\mathbf{u}_{R}^{g}$, and the others $\mathbf{u}_{L}^{k}$ and $\mathbf{u}_{R}^{k}$ - marked "retained" on figure 3 .

$$
\mathbf{u}_{L}=\left\{\begin{array}{c}
\mathbf{u}_{L}^{k} \\
\mathbf{u}_{L}^{g}
\end{array}\right\}, \quad \mathbf{u}_{R}=\left\{\begin{array}{c}
\mathbf{u}_{R}^{k} \\
\mathbf{u}_{R}^{g}
\end{array}\right\}
$$

Let $\phi_{R}$ be the matrix of the modes of the element when one node of the right side is fixed, as shown in figure 4 .

[Figure 4 about here.]

$\Phi_{R}$ is the modal matrix corresponding to right nodes of the element - it is a part of matrix $\phi_{R}$. According to the Craig \& Bampton theory [4], displacements of these right nodes can be expressed as follows:

$$
\mathbf{u}_{R}^{g}=\boldsymbol{\Phi}_{R} \mathbf{q}_{R}+\boldsymbol{\Psi}_{R} \mathbf{u}_{R}^{k}
$$

where $\boldsymbol{\Psi}_{R}$ is the matrix of the static modes, corresponding to the rigid body modes of the right side of the element.

Analogous matrices are defined for the left side of the element. Let $\phi_{L}$ be the matrix of the modes of the element when one node of the left side is fixed. $\boldsymbol{\Phi}_{L}$ is the modal matrix corresponding to left nodes of the element. Displacements of these left nodes can be expressed as follows:

$$
\mathbf{u}_{L}^{g}=\boldsymbol{\Phi}_{L} \mathbf{q}_{L}+\boldsymbol{\Psi}_{L} \mathbf{u}_{L}^{k}
$$

Thus, displacements $\mathbf{u}$ can be expressed as a function of generalized degrees of freedom $\mathbf{q}_{L}$ and $\mathbf{q}_{R}$ :

$$
\left\{\begin{array}{c}
\mathbf{u}_{L}^{k} \\
\mathbf{u}_{L}^{g} \\
\mathbf{u}_{R}^{k} \\
\mathbf{u}_{R}^{g}
\end{array}\right\}=\left[\begin{array}{cccc}
\mathbf{I} & \mathbf{0} & \mathbf{0} & \mathbf{0} \\
\boldsymbol{\Psi}_{L} & \mathbf{\Phi}_{L} & \mathbf{0} & \mathbf{0} \\
\mathbf{0} & \mathbf{0} & \mathbf{I} & \mathbf{0} \\
\mathbf{0} & \mathbf{0} & \boldsymbol{\Psi}_{R} & \mathbf{\Phi}_{R}
\end{array}\right]\left\{\begin{array}{l}
\mathbf{u}_{L}^{k} \\
\mathbf{q}_{L} \\
\mathbf{u}_{R}^{k} \\
\mathbf{q}_{R}^{k}
\end{array}\right\}
$$

Notice that as left degrees of freedom do not depend on right modes, and right degrees of freedom do not depend on left modes, assembling modal elements will be possible.

In order to assemble two elements, let write the relations between the nodal degrees of freedom of the left and right side of two elements. Degrees of freedom of the right side of the first element, called $\mathbf{u}_{R 1}^{k}$ and $\mathbf{u}_{R 1}^{g}$, must match with degrees of freedom of the left side of the second element, called $\mathbf{u}_{L 2}^{k}$ and $\mathbf{u}_{L 2}^{g}$ :

$$
\left\{\begin{array}{l}
\mathbf{u}_{R 1}^{k}=\mathbf{u}_{L 2}^{k} \\
\mathbf{u}_{R 1}^{g}=\mathbf{u}_{L 2}^{g}
\end{array}\right.
$$

Equation 6 can be written for elements $n$ and $n+1$ :

$$
\left\{\begin{array}{l}
\mathbf{u}_{R_{n}}^{k}=\mathbf{u}_{L_{n+1}}^{k} \\
\mathbf{u}_{R_{n}}^{g}=\mathbf{u}_{L_{n+1}}^{g}
\end{array}\right.
$$


Equations 6 leads to the expression of degrees of freedom of the left part of the second element as a function of degrees of freedom of the first element:

$$
\left\{\begin{array}{l}
\mathbf{u}_{L 2}^{k}=\mathbf{u}_{R 1}^{k} \\
\mathbf{q}_{L 2}=\tilde{\mathbf{\Phi}}_{L}\left(\mathbf{\Phi}_{R} \mathbf{q}_{R 1}+\left(\boldsymbol{\Psi}_{R}-\mathbf{\Psi}_{L}\right) \mathbf{u}_{R 1}^{k}\right)
\end{array}\right.
$$

which can be written for elements $n$ and $n+1$ :

$$
\left\{\begin{array}{l}
\mathbf{u}_{L_{n+1}}^{k}=\mathbf{u}_{R_{n}}^{k} \\
\mathbf{q}_{L_{n+1}}=\tilde{\mathbf{\Phi}}_{L}\left(\mathbf{\Phi}_{R} \mathbf{q}_{R_{n}}+\left(\boldsymbol{\Psi}_{R}-\mathbf{\Psi}_{L}\right) \mathbf{u}_{R_{n}}^{k}\right)
\end{array}\right.
$$

where $\tilde{\boldsymbol{\Phi}}_{L}$ is a pseudo-inverse matrix of $\boldsymbol{\Phi}_{L}$ :

$$
\tilde{\boldsymbol{\Phi}}_{L}=\left(\boldsymbol{\Phi}_{L}^{T} \boldsymbol{\Phi}_{L}\right)^{-1} \boldsymbol{\Phi}_{L}^{T}
$$

Equations 8 allow the assembling of $N$ elements, using transfer matrices $\mathbf{T}_{n}$ :

$$
\begin{aligned}
\mathbf{K}_{\mathrm{tot}} & =\sum_{n=1}^{N} \mathbf{T}_{n}^{T} \mathbf{K} \mathbf{T}_{n} \\
\mathbf{M}_{\mathrm{tot}} & =\sum_{n=1}^{N} \mathbf{T}_{n}^{T} \mathbf{M} \mathbf{T}_{n}
\end{aligned}
$$

Plates are assembled with the skeleton of the structure through the nodal degrees of freedom remaining from the modal analysis of the hollow parts of the structure. In this paper, only one plate will be used, located on the top of the structure. The entire structure is then analyzed using the "double modal synthesis" method proposed by Jezequel [5, 6]. This method uses "branch modes" to describe the behaviour of the boundaries between substructures. In this paper, will used these "branch modes" to discribe the behaviour of the skeleton of the structure.

Thus, mass and stiffness matrices of the structure can be split into degrees of freedom concerning plates and degrees of freedom concerning hollow parts of the structure. Degrees of freedom concerning hollow parts include generalized degrees of freedom resulting from the modal analysis of the elements constituting the skeleton, that will be denoted $\mathbf{q}_{H c}$, and generalized degrees of freedom resulting from the double modal synthesis, which will be denoted $\mathbf{q}_{H b}$. We denote $\mathbf{q}_{H}=\left\{\begin{array}{l}\mathbf{q}_{H c} \\ \mathbf{q}_{H b}\end{array}\right\}$. Vector $\mathbf{q}_{P}$ corresponds to the generalized degrees of freedom of the plates. Hence the motion equation:

$$
\left(-\omega^{2}\left[\begin{array}{ll}
\mathbf{M}_{H H} & \mathbf{M}_{H P} \\
\mathbf{M}_{P H} & \mathbf{M}_{P P}
\end{array}\right]+\left[\begin{array}{ll}
\mathbf{K}_{H H} & \mathbf{K}_{H P} \\
\mathbf{K}_{P H} & \mathbf{K}_{P P}
\end{array}\right]\right)\left\{\begin{array}{l}
\mathbf{q}_{H} \\
\mathbf{q}_{P}
\end{array}\right\}=\left\{\begin{array}{l}
\overline{\mathbf{f}}_{H} \\
\overline{\mathbf{f}}_{P}
\end{array}\right\}
$$

Generalized degrees of freedom used in the motion equation 13 are linked to nodal degrees of freedom through the following equations:

$$
\left\{\begin{array}{l}
\mathbf{u}_{P}=\boldsymbol{\Phi}_{P} \mathbf{q}_{P}+\mathbf{\Psi}_{P} \mathbf{u}_{H b} \\
\mathbf{u}_{H c}=\boldsymbol{\Phi}_{H c} \mathbf{q}_{H c}+\mathbf{\Psi}_{H c} \mathbf{u}_{H b} \\
\mathbf{u}_{H b}=\boldsymbol{\Phi}_{H b} \mathbf{q}_{H b}
\end{array}\right.
$$


where $\mathbf{u}_{P}$ are the nodal displacements of the plates, $\mathbf{u}_{H c}$ are the nodal displacements of the hollow parts corresponding to the generalized degrees of freedom $\mathbf{q}_{H c}$, and $\mathbf{u}_{\mathrm{Hb}}$ are the nodal displacements on the boundaries, associated to the branch modes.

Matrix $\boldsymbol{\Phi}_{P}$ is the modal matrix of the fixed modes of the plates. $\boldsymbol{\Psi}_{P}$ is the matrix of the static modes of the plates, as in the Craig \& Bampton method [4].

Matrices $\boldsymbol{\Phi}_{H c}$ and $\boldsymbol{\Psi}_{H c}$ are modal matrices resulting from the analysis of the hollow part described in [3].

Matrix $\boldsymbol{\Phi}_{H b}$ is the matrix of the "branch" modes of the structure [5, 6].

\subsection{Obtaining effective modal parameters}

Effective modal parameters used by P. Lemerle [1] are parameters that link degrees of freedom that are submitted to a displacement - excited degrees of freedom - and degrees of freedom whose displacements are to be minimized.

In order to obtain the effective modal parameters, we will use the modal analysis method proposed in section 2.1, but we will separate the degrees of freedom that will be excited - these degrees of freedom will remain nodal degrees of freedom.

In this paper, the excitations will concern some of the degrees of freedom we denoted $\mathbf{u}_{H b}$. These degrees of freedom will be called $\mathbf{u}_{H e}$. Hence equation 15 , coming from equation 14

$$
\left\{\begin{array}{l}
\mathbf{u}_{P}=\boldsymbol{\Phi}_{P} \mathbf{q}_{P}+\mathbf{\Psi}_{P} \mathbf{u}_{H b}+\boldsymbol{\Psi}_{P e} \mathbf{u}_{H e} \\
\mathbf{u}_{H c}=\boldsymbol{\Phi}_{H c} \mathbf{q}_{H c}+\boldsymbol{\Psi}_{H c} \mathbf{u}_{H b}+\boldsymbol{\Psi}_{H e} \mathbf{u}_{H e} \\
\mathbf{u}_{H b}=\boldsymbol{\Phi}_{H b} \mathbf{q}_{H b}
\end{array}\right.
$$

Thanks to the orthogonal properties of the modes used in the modal analysis, equation 13 can be written as follows, considering the damping matrix which we assume to be diagonal. Indeed, we use the modal damping assumption (also called Basile's hypothesis in French terminology), hence the following equation:

$$
\begin{aligned}
\left(-\omega^{2}\right. & {\left[\begin{array}{cccc}
\mathbf{M}_{E E} & \mathbf{M}_{E H c} & \mathbf{M}_{E H b} & \mathbf{M}_{E P} \\
\mathbf{M}_{H c E} & \mathbf{M}_{H c H c} & \mathbf{M}_{H c H b} & \mathbf{M}_{H c P} \\
\mathbf{M}_{H b E} & \mathbf{M}_{H b H c} & \mathbf{m}_{H b k} & \mathbf{M}_{H b P} \\
\mathbf{M}_{P E} & \mathbf{M}_{P H c} & \mathbf{M}_{P H b} & \mathbf{m}_{P k}
\end{array}\right]+i \omega\left[\begin{array}{cccc}
\mathbf{0} & \mathbf{0} & \mathbf{0} & \mathbf{0} \\
\mathbf{0} & \mathbf{c}_{H c k} & \mathbf{0} & \mathbf{0} \\
\mathbf{0} & \mathbf{0} & \mathbf{c}_{H b k} & \mathbf{0} \\
\mathbf{0} & \mathbf{0} & \mathbf{0} & \mathbf{c}_{P k}
\end{array}\right] } \\
+ & {\left.\left[\begin{array}{cccc}
\mathbf{K}_{E E} & \mathbf{K}_{E H c} & \mathbf{K}_{E H b} & \mathbf{0} \\
\mathbf{K}_{H c E} & \mathbf{K}_{H c H c} & \mathbf{K}_{H c H b} & \mathbf{0} \\
\mathbf{K}_{H b E} & \mathbf{K}_{H b H c} & \mathbf{k}_{H b k} & \mathbf{0} \\
\mathbf{0} & \mathbf{0} & \mathbf{0} & \mathbf{k}_{P k}
\end{array}\right]\right)\left\{\begin{array}{l}
\mathbf{u}_{H e} \\
\mathbf{q}_{H c} \\
\mathbf{q}_{H b} \\
\mathbf{q}_{P}
\end{array}\right\}=\left\{\begin{array}{c}
\overline{\mathbf{f}}_{E} \\
\overline{\mathbf{f}}_{H c} \\
\overline{\mathbf{f}}_{H b} \\
\overline{\mathbf{f}}_{P}
\end{array}\right\} }
\end{aligned}
$$

where matrices $\left[\mathbf{m}_{H b k}\right],\left[\mathbf{m}_{P k}\right],\left[\mathbf{k}_{H b k}\right],\left[\mathbf{k}_{P k}\right],\left[\mathbf{c}_{H b k}\right],\left[\mathbf{c}_{P k}\right]$ and $\left[\mathbf{c}_{H c k}\right]$ are diagonal matrices.

The analysis of the role played by the structural damping is not the purpose of the paper, that is why we chose a very simple way to take it into account.

To obtain the effective modal parameters, $\mathbf{u}_{P}$ must be expressed as a function of $\mathbf{f}_{P}$ and $\mathbf{u}_{\mathrm{He}}$. Let us express one of the last lines of equation 16 . 


$$
\begin{aligned}
-\omega^{2}\left(\mathbf{M}_{P E}^{k} \mathbf{u}_{H e}+\mathbf{M}_{P H c}^{k} \mathbf{q}_{H c}+\mathbf{M}_{P H b}^{k} \mathbf{q}_{H b}\right. & \left.+\mathbf{m}_{P k} \mathbf{q}_{P}^{k}\right) \\
& +i \omega \mathbf{c}_{P k} \mathbf{q}_{P}^{k}+\mathbf{k}_{P k} \mathbf{q}_{P}^{k}=\overline{\mathbf{f}}_{P}^{k}
\end{aligned}
$$

$\mathbf{M}_{P E}^{k}, \mathbf{M}_{P H c}^{k}, \mathbf{M}_{P H b}^{k}$ are the $k^{\text {th }}$ lines of matrices $\mathbf{M}_{P E}, \mathbf{M}_{P H c}, \mathbf{M}_{P H b} . \overline{\mathbf{f}}_{P}$ can be expressed in function of $\mathbf{f}_{P}$ as follow:

$$
\overline{\mathbf{f}}_{P}=\boldsymbol{\Phi}_{P}^{T} \mathbf{f}_{P}
$$

Equation 17 becomes:

$$
\mathbf{q}_{P}^{k}=\frac{\boldsymbol{\Phi}_{P}^{k T} \mathbf{f}_{P}+\omega^{2}\left(\mathbf{M}_{P E}^{k} \mathbf{u}_{H e}+\mathbf{M}_{P H c}^{k} \mathbf{q}_{H c}+\mathbf{M}_{P H b}^{k} \mathbf{q}_{H b}\right)}{-\omega^{2} \mathbf{m}_{P k}+i \omega \mathbf{c}_{P k}+\mathbf{k}_{P k}}
$$

where $\boldsymbol{\Phi}_{P}^{k}$ is the $k^{\text {th }}$ column of $\boldsymbol{\Phi}_{P}$. Equation 19 becomes:

$$
\begin{aligned}
\mathbf{u}_{P}= & \sum_{k} \boldsymbol{\Phi}_{P}^{k} \mathbf{q}_{P k}+\boldsymbol{\Psi}_{P} \mathbf{u}_{H b}+\mathbf{\Psi}_{P e} \mathbf{u}_{H e} \\
= & \sum_{k}\left(\frac{\boldsymbol{\Phi}_{P}^{k} \boldsymbol{\Phi}_{P}^{k T}}{-\omega^{2} \mathbf{m}_{P k}+i \omega \mathbf{c}_{P k}+\mathbf{k}_{P k}}\right) \mathbf{f}_{P} \\
& +\left[\sum_{k}\left(\frac{\omega^{2} \boldsymbol{\Phi}_{P}^{k}\left(\mathbf{M}_{P E}^{k}-\tilde{\mathbf{M}}_{P H c}^{k} \mathbf{\Psi}_{H e}\right)}{-\omega^{2} \mathbf{m}_{P k}+i \omega \mathbf{c}_{P k}+\mathbf{k}_{P k}}\right)+\boldsymbol{\Psi}_{P e}\right] \mathbf{u}_{H e} \\
& +\left[\sum_{k}\left(\frac{\omega^{2} \boldsymbol{\Phi}_{P}^{k}\left(\tilde{\mathbf{M}}_{P H b}^{k}-\tilde{\mathbf{M}}_{P H c}^{k} \boldsymbol{\Psi}_{H c}\right)}{-\omega^{2} \mathbf{m}_{P k}+i \omega \mathbf{c}_{P k}+\mathbf{k}_{P k}}\right)+\mathbf{\Psi}_{P}\right] \mathbf{u}_{H b} \\
& +\sum_{k}\left(\frac{-\omega^{2} \boldsymbol{\Phi}_{P}^{k} \tilde{\mathbf{M}}_{P H c}^{k}}{-\omega^{2} \mathbf{m}_{P k}+i \omega \mathbf{c}_{P k}+\mathbf{k}_{P k}}\right) \mathbf{u}_{H c}
\end{aligned}
$$

where $\mathbf{M}_{P H b}^{k}=\tilde{\mathbf{M}}_{P H b}^{k} \boldsymbol{\Phi}_{H b}$ and $\mathbf{M}_{P H c}^{k}=\tilde{\mathbf{M}}_{P H c}^{k} \boldsymbol{\Phi}_{H c}$. Matrix $\tilde{\boldsymbol{\Phi}}_{H c}$ is a pseudoinverse matrix of $\boldsymbol{\Phi}_{H c}$.

Two effective modal parameters can be deduced from equation 20, First, the dynamic flexibility matrix $\mathbf{G}$ is given by:

$$
\mathbf{G}(\omega)=\sum_{k}\left(\frac{\boldsymbol{\Phi}_{P}^{k} \boldsymbol{\Phi}_{P}^{k T}}{-\omega^{2} \mathbf{m}_{P k}+i \omega \mathbf{c}_{P k}+\mathbf{k}_{P k}}\right)
$$

It corresponds to the relation between a force applied on a plate and the displacements it causes.

Secondly, ignoring the static terms corresponding to the boundaries, the transmissibility matrix $\mathbf{T}$ is given by: 


$$
\mathbf{T}(\omega)=\sum_{k}\left(\frac{\omega^{2} \boldsymbol{\Phi}_{P}^{k}\left(\mathbf{M}_{P E}^{k}-\tilde{\mathbf{M}}_{P H c}^{k} \boldsymbol{\Psi}_{H e}\right)}{-\omega^{2} \mathbf{m}_{P k}+i \omega \mathbf{c}_{P k}+\mathbf{k}_{P k}}\right)
$$

It is possible to take the static terms into account, but we are more interested in the modal sum $\sum_{k}()$, wich allows to analyze the contribution of each mode of the structure, hence the choice of the transmissibility matrix of equation 22 .

This equation is linked to the relation between the excitation and the displacements it causes on the plate. Note that the excitation is a displacement excitation. They can be rewritten using effective parameters $\tilde{\mathbf{G}}$ and $\tilde{\mathbf{T}}$ as follows:

$$
\begin{aligned}
& \mathbf{G}(\omega)=\sum_{k} \frac{1}{1-\left(\frac{\omega}{\omega_{k}}\right)^{2}+2 i \xi_{k} \frac{\omega}{\omega_{k}}} \tilde{\mathbf{G}}_{k} \\
& \mathbf{T}(\omega)=\sum_{k} \frac{\left(\frac{\omega}{\omega_{k}}\right)^{2}}{1-\left(\frac{\omega}{\omega_{k}}\right)^{2}+2 i \xi_{k} \frac{\omega}{\omega_{k}}} \tilde{\mathbf{T}}_{k}
\end{aligned}
$$

where

$$
\begin{aligned}
\tilde{\mathbf{G}}_{k} & =\frac{\boldsymbol{\Phi}_{P}^{k} \boldsymbol{\Phi}_{P}^{k T}}{\omega_{k}^{2} \mathbf{m}_{P k}} \\
\tilde{\mathbf{T}}_{k} & =\frac{\boldsymbol{\Phi}_{P}^{k}\left(\mathbf{M}_{P E}^{k}-\tilde{\mathbf{M}}_{P H c}^{k} \mathbf{\Psi}_{H e}\right)}{\mathbf{m}_{P k}}
\end{aligned}
$$

with the notations $\mathbf{c}_{P k}=2 \xi_{k} \sqrt{\mathbf{k}_{P k} \mathbf{m}_{P k}}$ and $\omega_{k}=\sqrt{\frac{\mathbf{k}_{P_{k}}}{\mathbf{m}_{P k}}}$. Matrices $\tilde{\mathbf{G}}_{k}$ and $\tilde{\mathbf{T}}_{k}$ are called effective modal parameters.

\section{Optimization based on effective modal parameters}

\subsection{Criteria used for optimization}

In this section, we will deduce criteria from the flexibility and transmissibility matrices proposed in section 2.2. The sums $\sum_{k}()$ that appear in these matrices correspond to a superposition of modes. Thus, optimization criteria can be written as follow:

$$
\begin{aligned}
& C_{G}=\max _{k}\left|\frac{\boldsymbol{\Phi}_{P}^{k} \boldsymbol{\Phi}_{P}^{k T}}{\omega_{k}^{2} \mathbf{m}_{P k}}\right| \\
& C_{T}=\max _{k}\left|\frac{\boldsymbol{\Phi}_{P}^{k}\left(\mathbf{M}_{P E}^{k}-\tilde{\mathbf{M}}_{P H c}^{k} \mathbf{\Psi}_{H e}\right)}{\mathbf{m}_{P k}}\right|
\end{aligned}
$$


where the norm $|\mathbf{x}|$ is the maximal component of matrix $\mathbf{x}$. Considering these criteria, it is possible to optimize the structure. Moreover, obtaining the value $k_{\max }$ allows us to understand which mode is responsible for the value of the criteria.

\subsection{Methods used for optimization}

We will show examples using the criteria we propose through a two level strategy for optimization. First, a genetic algorithm will be used. Secondly, a local optimization will be processed in order to obtain more precise results.

\subsubsection{Genetic optimization}

Genetic optimization is often used in the case of multi-objective optimization problems. The aim of this section is to generate a Pareto front in order to obtain various optimized solutions for the optimization problem. Overviews of multi-objective optimization genetic algorithms can be found in [7, 8, 9]. The genetic algorithm that will be used in this section is quite simple. Let $\alpha_{i}$ be the $p$ parameters to optimize. $\mathbf{A}_{1}$ is a first matrix of $N$ sets of parameters:

$$
\mathbf{A}_{1}=\left[\begin{array}{ccccc}
\alpha_{1}^{1} & \alpha_{2}^{1} & \cdots & \alpha_{p-1}^{1} & \alpha_{p}^{1} \\
\alpha_{1}^{2} & \alpha_{2}^{2} & \cdots & \alpha_{p-1}^{2} & \alpha_{p}^{2} \\
\vdots & \vdots & \ddots & \vdots & \vdots \\
\alpha_{1}^{N-1} & \alpha_{2}^{N-1} & \cdots & \alpha_{p-1}^{N-1} & \alpha_{p}^{N-1} \\
\alpha_{1}^{N} & \alpha_{2}^{N} & \cdots & \alpha_{p-1}^{N} & \alpha_{p}^{N}
\end{array}\right]
$$

The lines of matrix $A_{1}$ are the $N$ sets of parameters chosen for the first iteration of the algorithm. The values of the criteria we propose are computed using these sets of parameters. The mass of the structure can also be a third criterion, for the structure mustn't become too heavy. A set of $\alpha_{i}$ is then selected if no other set gives better results on both criteria - and the mass. The other sets of $\alpha_{i}$ are combined in order to create another matrix $\mathbf{A}_{2}$ of $N$ sets of parameters that will include the selected sets. Figure 5 sums up the algorithm used for this optimization.

[Figure 5 about here.]

Once the optimal sets of $\alpha_{i}$ have been found, the results can be plotted on a pareto diagram, which will be done bellow. Various "optimal solutions" are found, and the local optimization can be made from one of these "optimal solutions".

\subsubsection{Local optimization}

The genetic algorithm proposed in section 3.2.1 gives various results, but the best value for each $\alpha_{i}$ is not precisely determined. In order to obtain more accurate results, we propose a local method.

Three criteria are to be considered for the optimization. $C_{G}$ and $C_{T}$ have been defined in section 2.2. The third criterion will be the mass of the structure, denoted $C_{M}$. 
The optimization problem can then be written:

$$
\text { Minimize } C_{M}(\boldsymbol{\alpha}) \text { assuming }\left\{\begin{array}{l}
\tilde{C}_{G}(\boldsymbol{\alpha}) \leq 0 \\
\tilde{C}_{T}(\boldsymbol{\alpha}) \leq 0
\end{array}\right.
$$

where $\boldsymbol{\alpha}=\left[\alpha_{1}, \alpha_{2}, \ldots, \alpha_{p}\right] . \tilde{C}_{G}$ and $\tilde{C}_{T}$ are directly linked with $C_{G}$ and $C_{T}$. The method used in this paper requires that the criteria be derivated, which is not possible with $C_{G}$ and $C_{T}$. Such criteria are defined as follow:

$$
\begin{aligned}
& \tilde{C}_{G}=\frac{1}{4} \log \sum_{k}\left(\frac{\boldsymbol{\Phi}_{P}^{k} \boldsymbol{\Phi}_{P}^{k T}}{-\omega_{k}^{2} \mathbf{m}_{P k}}\right)^{4}-c_{g} \\
& \tilde{C}_{T}=\frac{1}{4} \log \sum_{k}\left(\frac{\boldsymbol{\Phi}_{P}^{k}\left(\mathbf{M}_{P E}^{k}-\tilde{\mathbf{M}}_{P H c}^{k} \boldsymbol{\Psi}_{H e}\right)}{\mathbf{m}_{P k}}\right)^{4}-c_{t}
\end{aligned}
$$

where $c_{g}$ and $c_{t}$ are the objectives of the criteria.

$\tilde{C}_{G}$ and $\tilde{C}_{T}$ have almost the same minima and extrema as $C_{G}$ and $C_{T}$. To prove this, let us consider the function $f_{p}$ as follows:

$$
f_{p}: \quad x \longrightarrow \sqrt[p]{a_{1}(x)^{p}+a_{2}(x)^{p}+\cdots+a_{n}(x)^{p}}
$$

where $a_{q}(x)>0 \quad \forall q \leq n \forall x$. It is possible to write:

$$
\lim _{p \rightarrow+\infty} f_{p}(x)=\max \left[a_{1}(x), a_{2}(x), \ldots, a_{n}(x)\right]
$$

Indeed:

$$
\begin{array}{r}
\sqrt[p]{\max \left[a_{1}(x), a_{2}(x), \ldots, a_{n}(x)\right]^{p}}<f_{p}(x)<\sqrt[p]{n \max \left[a_{1}(x), a_{2}(x), \ldots, a_{n}(x)\right]^{p}} \\
\forall p \in \mathbb{N} .
\end{array}
$$

Equation 35 leads to:

$$
\begin{array}{r}
\max \left[a_{1}(x), a_{2}(x), \ldots, a_{n}(x)\right]<f_{p}(x)<\sqrt[p]{n} \max \left[a_{1}(x), a_{2}(x), \ldots, a_{n}(x)\right] \\
\forall p \in \mathbb{N} .
\end{array}
$$

As $\lim _{p \rightarrow+\infty} \sqrt[p]{n}=1$, we can deduce $\lim _{p \rightarrow+\infty} f_{p}(x)=\max \left[a_{1}(x), a_{2}(x), \ldots, a_{n}(x)\right]$. Thus, if $p$ is high enough, $\tilde{C}_{G}$ and $\tilde{C}_{T}$ have almost the same minima and extrema as $C_{G}$ and $C_{T}$. In this section, we will use $\tilde{C}_{G}$ and $\tilde{C}_{T}$ instead of $C_{G}$ and $C_{T}$, for these criteria may be derivated, whereas $C_{G}$ and $C_{T}$ cannot.

The optimization method we use in this paper is based on the Kühn \& Tucker conditions, which can be written as follow: 


$$
\begin{array}{r}
\exists\left(\lambda_{1}(\boldsymbol{\alpha}), \lambda_{2}(\boldsymbol{\alpha})\right), \quad \lambda_{1}(\boldsymbol{\alpha}) \nabla \tilde{C}_{G}(\boldsymbol{\alpha})+\lambda_{2}(\boldsymbol{\alpha}) \nabla \tilde{C}_{T}(\boldsymbol{\alpha})+\nabla C_{M}(\boldsymbol{\alpha})=0 \\
\text { and }\left\{\begin{array}{l}
\lambda_{1}(\boldsymbol{\alpha}) \geq 0 \\
\lambda_{2}(\boldsymbol{\alpha}) \geq 0
\end{array}\right.
\end{array}
$$

The problem we propose requires equation 37 to be written as follows:

$$
\forall i=1,2,3, \ldots, p \quad \lambda_{1}(\boldsymbol{\alpha}) \frac{\frac{\partial \tilde{C}_{G}}{\partial \alpha_{i}}(\boldsymbol{\alpha})}{\frac{\partial C_{M}}{\partial \alpha_{i}}(\boldsymbol{\alpha})}+\lambda_{2}(\boldsymbol{\alpha}) \frac{\frac{\partial \tilde{C}_{T}}{\partial \alpha_{i}}(\boldsymbol{\alpha})}{\frac{\partial C_{M}}{\partial \alpha_{i}}(\boldsymbol{\alpha})}=-1
$$

In this equation, we assume $\frac{\partial C_{M}}{\partial \alpha_{i}}(\boldsymbol{\alpha}) \neq 0$. A relaxation parameter $\gamma$ is then introduced. Equation 38 becomes:

$$
\alpha_{i}=\gamma \alpha_{i}-\left[(1-\gamma)\left(\lambda_{1}(\boldsymbol{\alpha}) \frac{\frac{\partial \tilde{C}_{G}}{\partial \alpha_{i}}(\boldsymbol{\alpha})}{\frac{\partial C_{M}}{\partial \alpha_{i}}(\boldsymbol{\alpha})}+\lambda_{2}(\boldsymbol{\alpha}) \frac{\frac{\partial \tilde{C}_{T}}{\partial \alpha_{i}}(\boldsymbol{\alpha})}{\frac{\partial C_{M}}{\partial \alpha_{i}}(\boldsymbol{\alpha})}\right)\right] \alpha_{i}
$$

This equation leads to the following recurrence equation:

$$
\alpha_{i}^{k+1}=\gamma \alpha_{i}^{k}-\left[(1-\gamma)\left(\left.\lambda_{1}(\boldsymbol{\alpha}) \frac{\frac{\partial \tilde{C}_{G}}{\partial \alpha_{i}}(\boldsymbol{\alpha})}{\frac{\partial C_{M}}{\partial \alpha_{i}}(\boldsymbol{\alpha})}\right|_{\alpha_{i}=\alpha_{i}^{k}}+\left.\lambda_{2}(\boldsymbol{\alpha}) \frac{\frac{\partial \tilde{C}_{T}}{\partial \alpha_{i}}(\boldsymbol{\alpha})}{\frac{\partial C_{M}}{\partial \alpha_{i}}(\boldsymbol{\alpha})}\right|_{\alpha_{i}=\alpha_{i}^{k}}\right)\right] \alpha_{i}^{k}
$$

This method is similar to the gradient method, but the descent direction here is:

$$
d_{k}=-(1-\gamma) R_{i}(\boldsymbol{\alpha}) \alpha_{i}^{k}
$$

where:

$$
R_{i}(\boldsymbol{\alpha})=1+\left.\lambda_{1}(\boldsymbol{\alpha}) \frac{\frac{\partial \tilde{C}_{G}}{\partial \alpha_{i}}(\boldsymbol{\alpha})}{\frac{\partial C_{M}}{\partial \alpha_{i}}(\boldsymbol{\alpha})}\right|_{\alpha_{i}=\alpha_{i}^{k}}+\left.\lambda_{2}(\boldsymbol{\alpha}) \frac{\frac{\partial \tilde{C}_{T}}{\partial \alpha_{i}}(\boldsymbol{\alpha})}{\frac{\partial C_{M}}{\partial \alpha_{i}}(\boldsymbol{\alpha})}\right|_{\alpha_{i}=\alpha_{i}^{k}}
$$

The convergence condition can then be written as for the gradient method. $\tilde{R}: \boldsymbol{\alpha} \longrightarrow\left\{R_{i}(\boldsymbol{\alpha}) \alpha_{i}\right\}$ must satisfy the following equations:

$$
\begin{array}{lll}
\exists \kappa>0 & \forall u, v & \langle\tilde{R}(v)-\tilde{R}(u), v-u\rangle \geq \kappa\|v-u\|^{2} \\
\exists \xi>0 & \forall u, v & \|\tilde{R}(v)-\tilde{R}(u)\| \leq \xi\|v-u\|
\end{array}
$$

It is then possible to find a value for $\gamma$. The algorithm converges if the following relation is satisfied:

$$
1-\frac{2 \kappa}{\xi^{2}}<\gamma<1
$$


$\gamma$ must be chosen close to 1 if we want to be sure that the algorithm converges. The smaller $\gamma$ is, the faster the algorithm will converge, but it may not converge at all if $\gamma$ is too small...

For each step of the algorithm, $\lambda_{i}(\boldsymbol{\alpha})$ is being computed. The method used to obtain these $\lambda_{i}(\boldsymbol{\alpha})$ is now explained. Let us take $\Delta \alpha_{i}$, such as:

$$
\left\{\begin{array}{l}
\tilde{C}_{G}\left(\alpha_{1}+\Delta \alpha_{1}, \alpha_{2}+\Delta \alpha_{2}, \ldots, \alpha_{p}+\Delta \alpha_{p}\right)=0 \\
\tilde{C}_{T}\left(\alpha_{1}+\Delta \alpha_{1}, \alpha_{2}+\Delta \alpha_{2}, \ldots, \alpha_{p}+\Delta \alpha_{p}\right)=0
\end{array}\right.
$$

This equation allows us to express $\Delta \tilde{C}_{G}\left(\alpha_{1}, \alpha_{2}, \ldots, \alpha_{p}\right)$ as follows:

$$
\begin{aligned}
\Delta \tilde{C}_{G}\left(\alpha_{1}, \alpha_{2}, \ldots, \alpha_{p}\right) & =\tilde{C}_{G}\left(\alpha_{1}+\Delta \alpha_{1}, \alpha_{2}+\Delta \alpha_{2}, \ldots, \alpha_{p}+\Delta \alpha_{p}\right)-\tilde{C}_{G}\left(\alpha_{1}, \alpha_{2}, \ldots, \alpha_{p}\right) \\
& =-\tilde{C}_{G}\left(\alpha_{1}, \alpha_{2}, \ldots, \alpha_{p}\right) \\
& =\sum_{i=1}^{p} \frac{\partial \tilde{C}_{G}}{\partial \alpha_{i}} \Delta \alpha_{i}
\end{aligned}
$$

Replacing $\Delta \alpha_{i}$ in equation $47, \tilde{C}_{G}$ can be written as follows:

$$
\tilde{C}_{G}\left(\alpha_{1}, \alpha_{2}, \ldots, \alpha_{p}\right)=(1-\gamma) \sum_{i=1}^{p} \frac{\partial \tilde{C}_{G}}{\partial \alpha_{i}}\left[1+\lambda_{1} \frac{\frac{\partial \tilde{C}_{G}}{\partial \alpha_{i}}}{\frac{\partial C_{M}}{\partial \alpha_{i}}}+\lambda_{2} \frac{\frac{\partial \tilde{C}_{T}}{\partial \alpha_{i}}}{\frac{\partial C_{M}}{\partial \alpha_{i}}}\right] \alpha_{i}^{k}
$$

In order to simplify the notations, we denote $\lambda_{1}=\lambda_{1}(\boldsymbol{\alpha}), \lambda_{1}=\lambda_{1}(\boldsymbol{\alpha})$ and $\frac{\partial \tilde{C}_{j}}{\partial \alpha_{i}}=\left.\frac{\partial \tilde{C}_{j}}{\partial \alpha_{i}}(\boldsymbol{\alpha})\right|_{\alpha_{i}=\alpha_{i}^{k}}$.

The same equation can be written for $\tilde{C}_{T}$, which allows us to write the following equation:

$$
\left[\begin{array}{cc}
\sum_{i=1}^{p} \frac{\left(\frac{\partial \tilde{C}_{G}}{\partial \alpha_{i}}\right)^{2}}{\frac{\partial C_{M}}{\partial \alpha_{i}}} \alpha_{i}^{k} & \sum_{i=1}^{p} \frac{\partial \tilde{C}_{G}}{\partial \alpha_{i}} \frac{\frac{\partial \tilde{C}_{T}}{\partial \alpha_{i}}}{\frac{\partial C_{M}}{\partial \alpha_{i}}} \alpha_{i}^{k} \\
\sum_{i=1}^{p} \frac{\partial \tilde{C}_{T}}{\partial \alpha_{i}} \frac{\frac{\partial \tilde{C}_{G}}{\partial \alpha_{i}}}{\frac{\partial C_{M}}{\partial \alpha_{i}}} \alpha_{i}^{k} & \sum_{i=1}^{p} \frac{\left(\frac{\partial \tilde{C}_{T}}{\partial \alpha_{i}}\right)^{2}}{\frac{\partial C_{M}}{\partial \alpha_{i}}} \alpha_{i}^{k}
\end{array}\right]\left\{\begin{array}{c}
\lambda_{1} \\
\lambda_{2}
\end{array}\right\}=\left\{\begin{array}{c}
\frac{\tilde{C}_{G}}{1-\gamma}-\sum_{i=1}^{p} \frac{\partial \tilde{C}_{G}}{\partial \alpha_{i}} \alpha_{i} \\
\frac{\tilde{C}_{T}}{1-\gamma}-\sum_{i=1}^{p} \frac{\partial \tilde{C}_{T}}{\partial \alpha_{i}} \alpha_{i}
\end{array}\right\}
$$

This equation allows us to obtain $\left(\lambda_{1}, \lambda_{2}\right)$ for each step of the optimization.

\section{Results}

The method proposed in this paper has been tested on a complex structure including hollow parts and plates. The parameters we choose to optimize are linked to the geometry of the hollow parts. Indeed, we will optimize $D$ and $\lambda$, as shown in figure 6. 
[Figure 6 about here.]

The optimization methods we will use in the next sections will use $D$ and $\lambda$ as parameters. The hollow parts of the structure shown in figure 1 are split into 8 parts, and each part is optimized with optimal values of $D$ and $\lambda$. Thus, 16 parameters are to be optimized.

During the optimization, matrices $\mathbf{M}$ and $\mathbf{K}$ are updated in order to take into account the evolution of the geometry of the hollow parts of the structure. Actually, modes concerning these hollow parts need to be re-computed for each iteration. However, only one element for each set of parameters has to be re-computed, thanks to the use of our substructuring method to describe the hollow parts of the structure. Parametric methods can also be used to solve numerical difficulties.

\subsection{Structure used for optimization}

The structure used for the optimization is given in figure 1 It is made of hollow parts and plates, that have been analyzed using the methods proposed in section 2.1 .

Because of the calculation costs, we chose a structure which is not too large, i.e. 2.5 meters long, as given in figure 1 .

\subsection{Analysis of the criteria}

The analysis of criteria $C_{G}$ and $C_{T}$ shows which modes are responsible for the displacements needing to be reduced. Figure 8 shows the values of $C_{G}^{k}$, which are part of criterion $C_{G}$ :

$$
C_{G}^{k}=\left|\frac{\boldsymbol{\Phi}_{P}^{k} \boldsymbol{\Phi}_{P}^{k T}}{\omega_{k}^{2} \mathbf{m}_{P k}}\right|
$$

The same analysis can be made for $C_{T}^{k}$ :

$$
C_{T}^{k}=\left|\frac{\boldsymbol{\Phi}_{P}^{k}\left(\mathbf{M}_{P E}^{k}-\tilde{\mathbf{M}}_{P H c}^{k} \mathbf{\Psi}_{H e}\right)}{\mathbf{m}_{P k}}\right|
$$

Figure 7 shows the values of $C_{T}^{k}$, which are part of criterion $C_{T}$.

[Figure 7 about here.]

[Figure 8 about here.]

Figures 7 and 8 show that criteria $C_{G}$ and $C_{T}$ do not necessarily depend on the same modes. In this example, we only analyze the criteria for the first 50 modes of the structure. Figure 7 shows that the $45^{\text {th }}$ mode is mostly responsible for the value of criterion $C_{G}$, whereas figure 8 shows that the $6^{\text {th }}, 7^{\text {th }}, 22^{\text {th }}$ and $23^{\text {th }}$ modes are responsible for the value of criterion $C_{T}$. Thus, it is necessary to take these two criteria into account to optimize the structure. 


\subsection{Results of genetic optimization}

In this section, we present the results obtained using the genetic algorithm given in figure 5. Figures 9, 10, 11 and 12 show the pareto diagrams of the sets $\mathbf{A}_{i}$. Units on $x$ and $y$ axis are not important because it depends on the values of $c_{g}$ and $c_{t}$. The excitation points are located on the hollow parts, whereas the displacements to be reduced are located on the plate on the top of the structure.

[Figure 9 about here.]

[Figure 10 about here.]

[Figure 11 about here.]

[Figure 12 about here.]

These results show that the algorithm is able to produce a set of optimal parameters. These optimal parameters form a curve including points that are the closest to the origin of the graphs. The pareto diagram is shown in $3 \mathrm{D}$, but because it is not easily understood, we have also shown three 2D diagrams.

Figure 13 shows the evolution of the number of pareto points as a function of the progress of the optimization. At the end of the algorithm, all points of the set $\mathbf{A}_{N}$ are "pareto points" - i.e. optimal points.

[Figure 13 about here.]

\subsection{Results of local optimization}

A local optimization is now processed, using the same structure and the same excitation points. Figure 14 shows the square speed of a point of the plate, whereas figure 15 shows the evolution of the criteria during the optimization.

[Figure 14 about here.]

[Figure 15 about here.]

In figure 15, the ratio between the criteria and their initial values is plotted. That is why the first values are 1 in the three cases. Criteria do not change much because the values chosen for the parameters $\alpha_{i}$ are close to the optimal values. This is a condition for the algorithm to work well. Notice that the algorithm is not always able to converge because some conditions on the parameters have been set. The parameters to be optimized must remain in an interval of acceptable values.

In figure 14, the optimized value for the square speed is not much better than the initial value because the parameters have not changed much. Nevertheless, this value is not bad and the mass of the structure has decreased, which is why the optimal solution is better than the initial one. 


\section{Conclusion}

The optimization method we propose in this paper is based on criteria that allow us to analyze the origin of the problems we want to solve - reducing noise in complex structures. These criteria have been used with two kinds of optimization algorithms and the results prove that they are quite efficient and able to represent th vibration level of the structure.

Examples given in this paper are based on an excitation located on a point of a hollow part. It is possible to do the same analysis based on another excitation. Criteria will then be different, but the results should be quite good. The algorithms proposed here are quite easy to use with the structures we chose.

\section{References}

[1] P. Lemerle, Optimisation des structures selon des critères imposés par la discrétion acoustique des navires, Phd thesis, École Centrale de Lyon (1994).

[2] W. Suweca, L. Jézéquel, Optimal structural design with damping constraint limitations, International journal for numerical methods in engineering 35 (1992) 21-35.

[3] S. Besset, L. Jezequel, Modal analysis of hollow parts of a structure, in: IMAC XXIV: A Conference \& Exposition on Structural Dynamics, 2005.

[4] R. R. Craig, M. C. C. Bampton, Coupling of substructures for dynamic analysis, AIAA Journal 6 (1968) 1313-1321.

[5] L. Jezequel, H. D. Setio, Component modal synthesis methods based on hybrid models, part i : Theory of hybrid models and modal truncation methods, ASME Journal of Applied Mechanics 61 (1994) 100-108.

[6] L. Jezequel, H. D. Setio, Component modal synthesis methods based on hybrid models, part ii : Numerical tests and experimental identification of hybrid model, ASME Journal of Applied Mechanics 61 (1994) 109-116.

[7] W. Stadler, A survey of multicriteria optimization, or the vector maximum problem, Journal of optimization theroy and application 29 (1979) 1-52.

[8] C. Fonseca, P. Fleming, An overview of evolutionary algorithms in multiobjective optimization, Evolutionary Computation 3 (1995) 1-18.

[9] D. E. Goldberg, Genetic Algorithms in Search, Optimization and Machine Learneing, Addison-Wesley, Reading, Massachusetts, 1989. 


\section{List of Figures}

$1 \quad$ Structure to be optimize . . . . . . . . . . . . . . . . 16

2 Hollow part to study . . . . . . . . . . . . . . . . . . . . . . . . . . 17

3 Element of the hollow part . . . . . . . . . . . . . . 18

4 Element of a hollow part . . . . . . . . . . . . . . . . 19

5 Algorithm used for genetic optimization . . . . . . . . . . . . 20

6 Hollow part included in the structure . . . . . . . . . . . . . . . 21

$7 \quad$ Values of $C_{C}^{k} \ldots \ldots \ldots \ldots 22$

$8 \quad$ Values of $C_{T}^{k} \ldots \ldots \ldots \ldots \ldots$

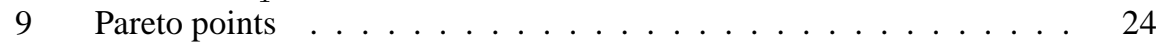

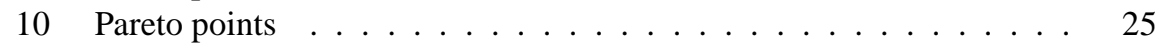

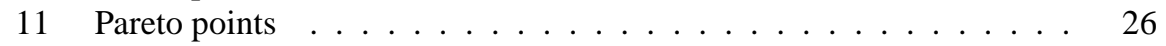

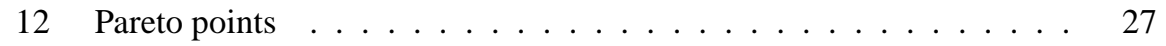

13 Evolution of the number of pareto points . . . . . . . . . 28

14 Square speed of a point of the plate $(-$ : Not optimized, . : : Optimized $) 29$

15 Evolution of the criteria $\left(-\right.$ : Mass, $\left.\ldots: \tilde{C}_{C},-\cdots: \tilde{C}_{T}\right) \ldots 30$ 


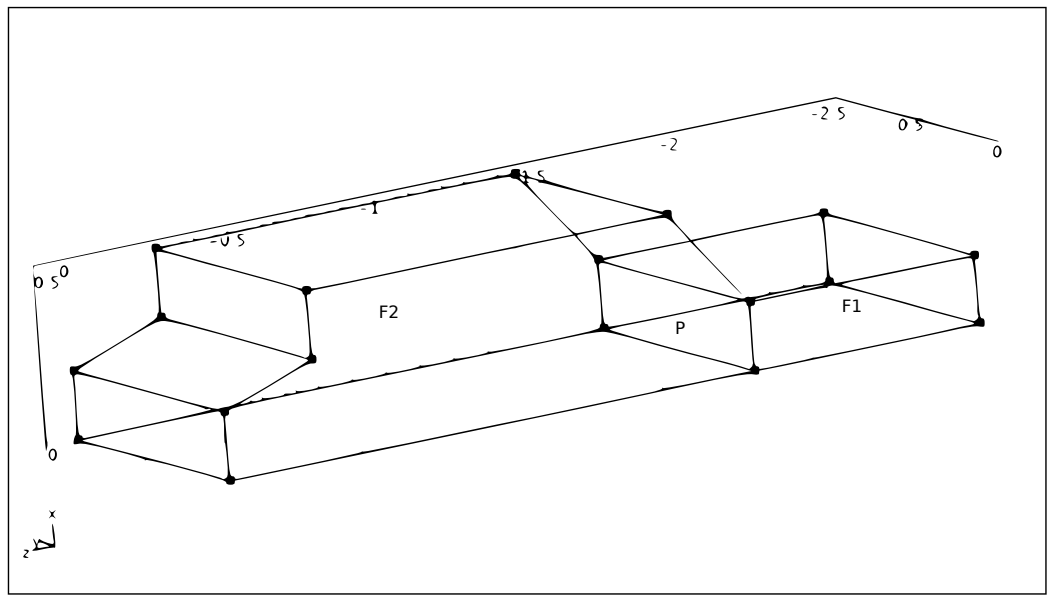

Figure 1: Structure to be optimize 


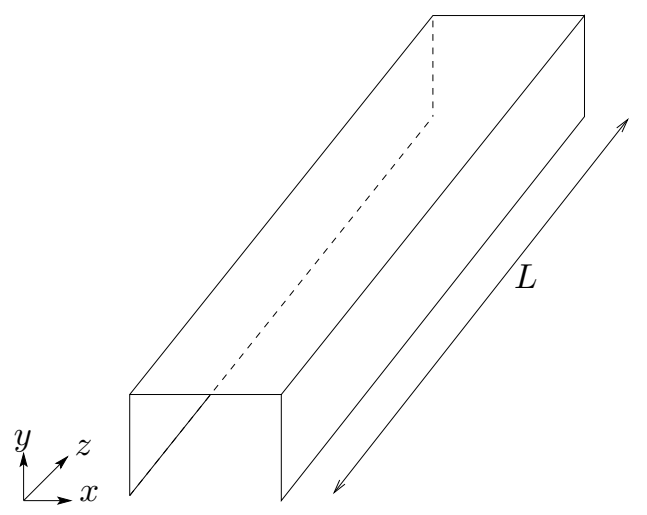

Figure 2: Hollow part to study 


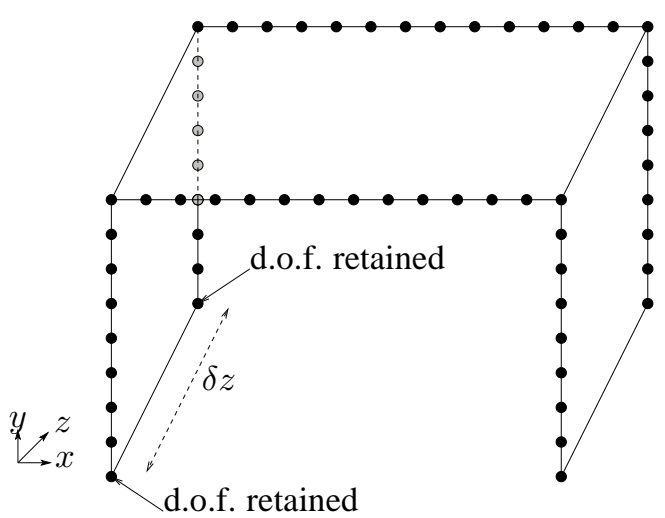

Figure 3: Element of the hollow part 


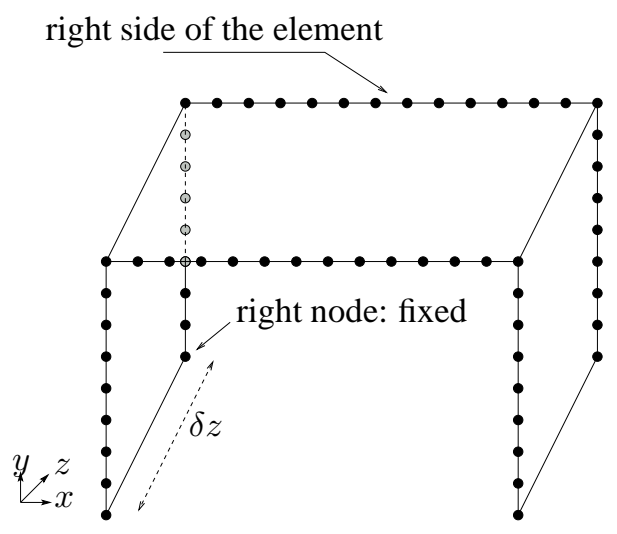

Figure 4: Element of a hollow part 


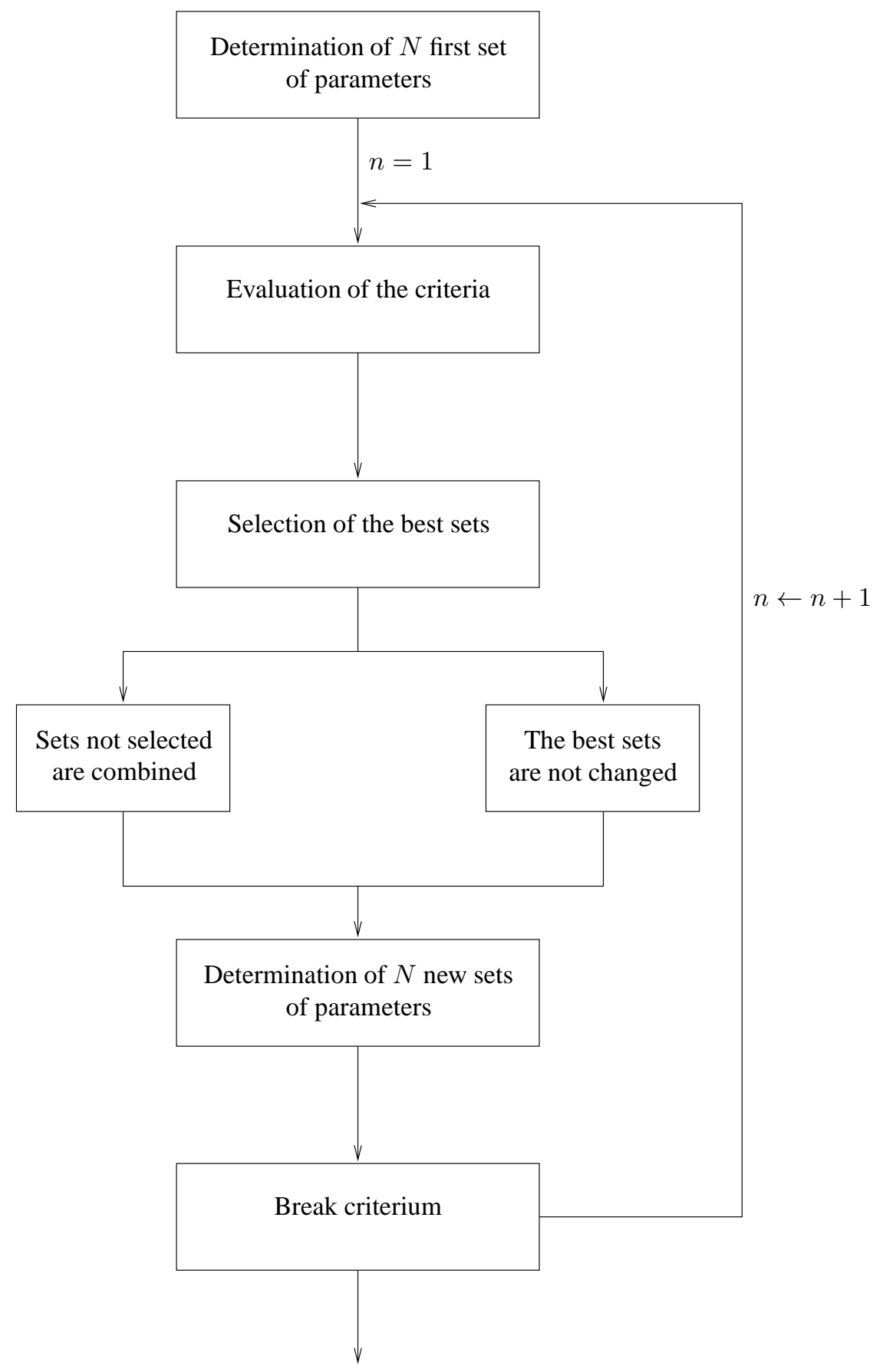

Figure 5: Algorithm used for genetic optimization 


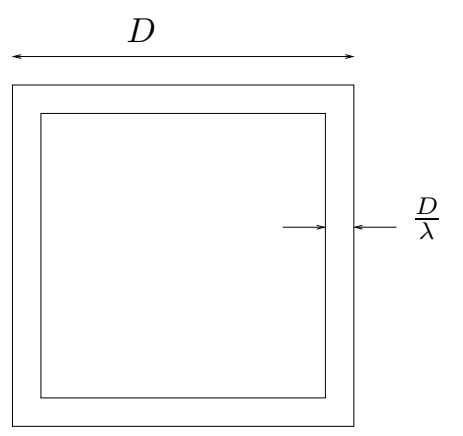

Figure 6: Hollow part included in the structure 


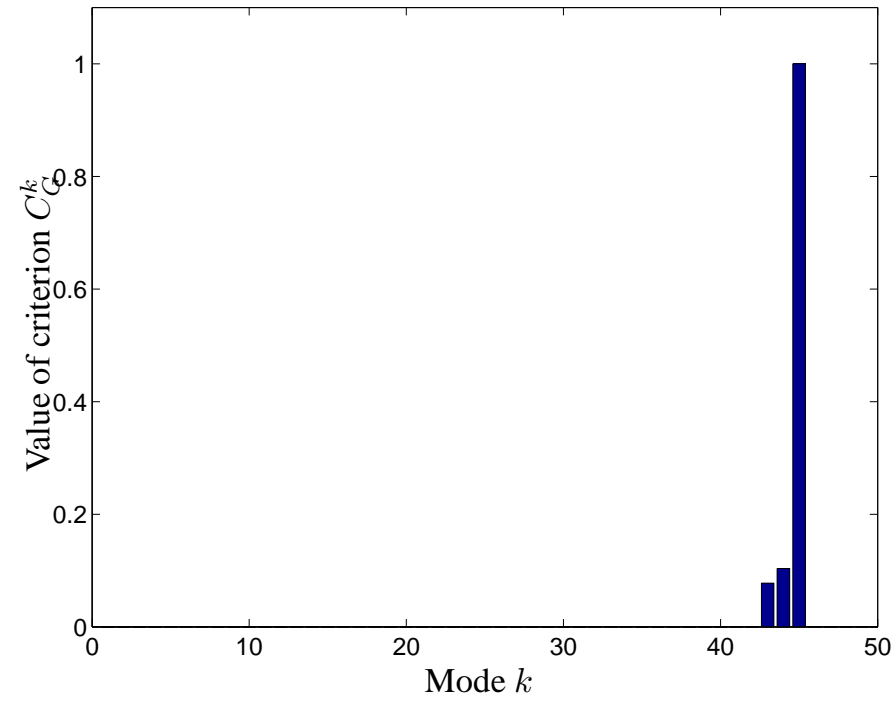

Figure 7: Values of $C_{G}^{k}$ 


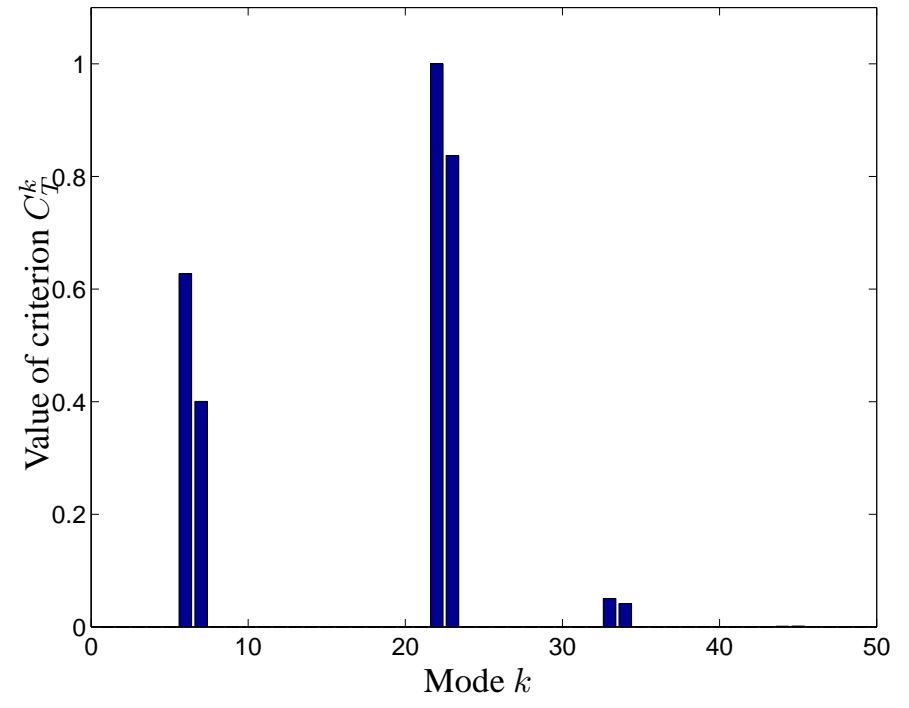

Figure 8: Values of $C_{T}^{k}$ 


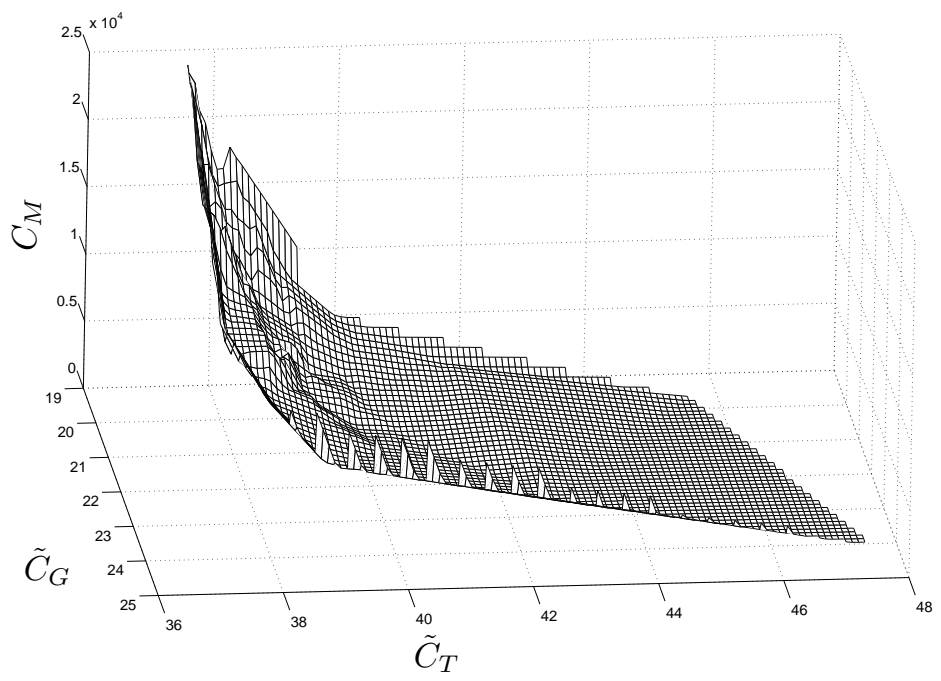

Figure 9: Pareto points 


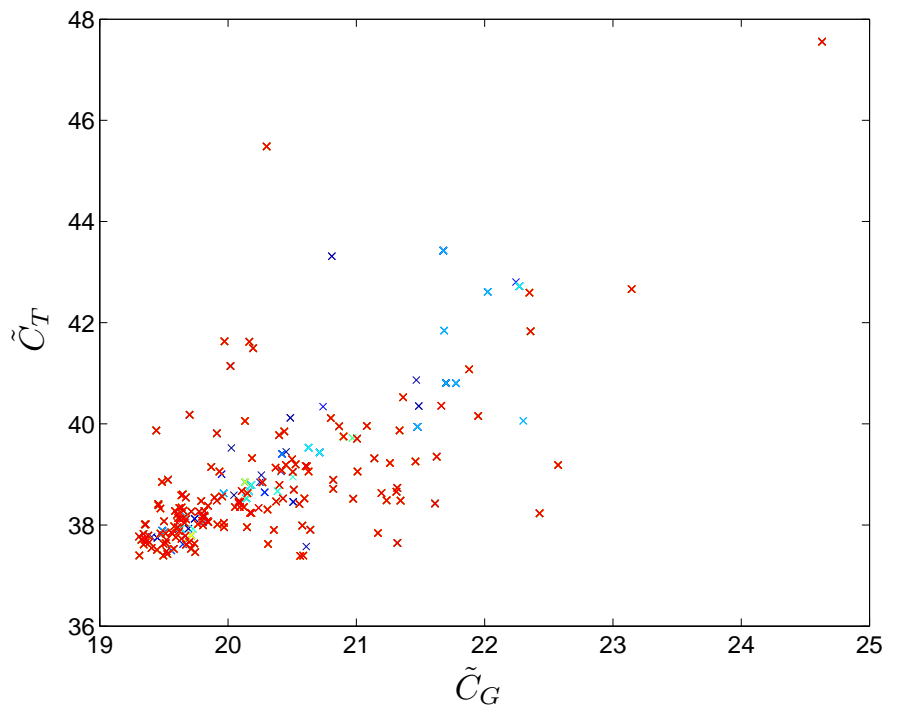

Figure 10: Pareto points 


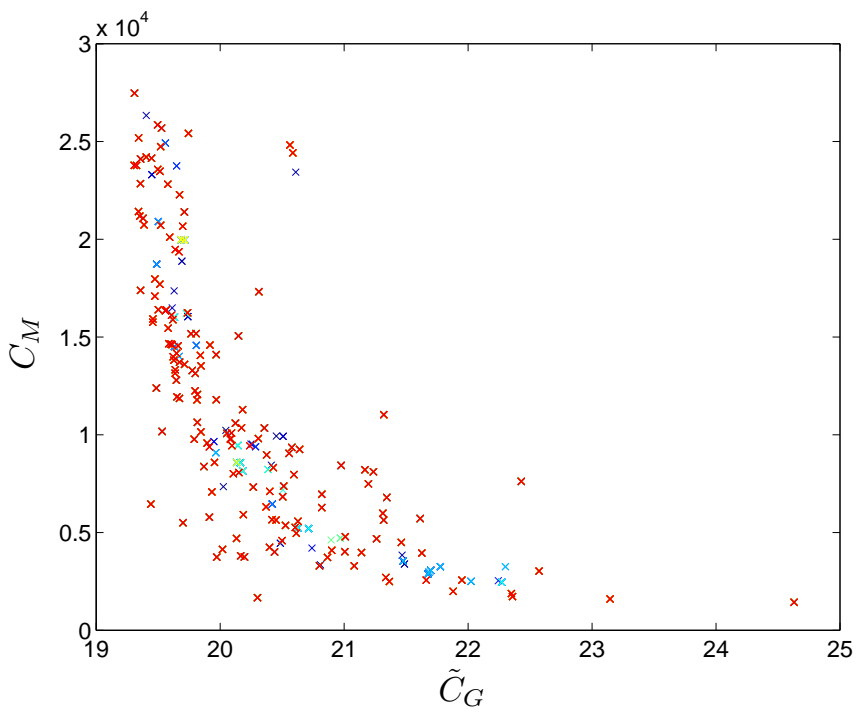

Figure 11: Pareto points 


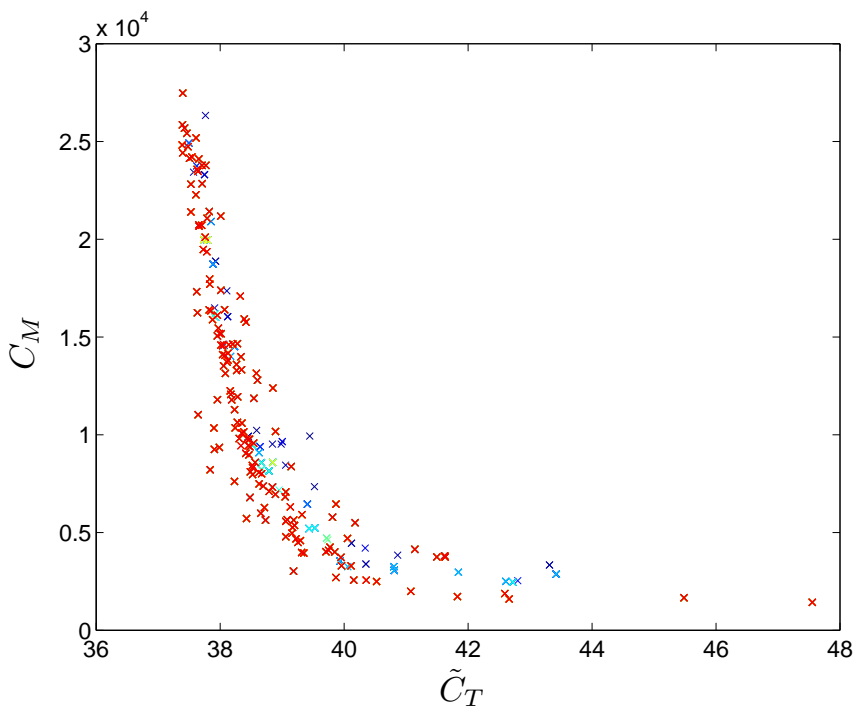

Figure 12: Pareto points 


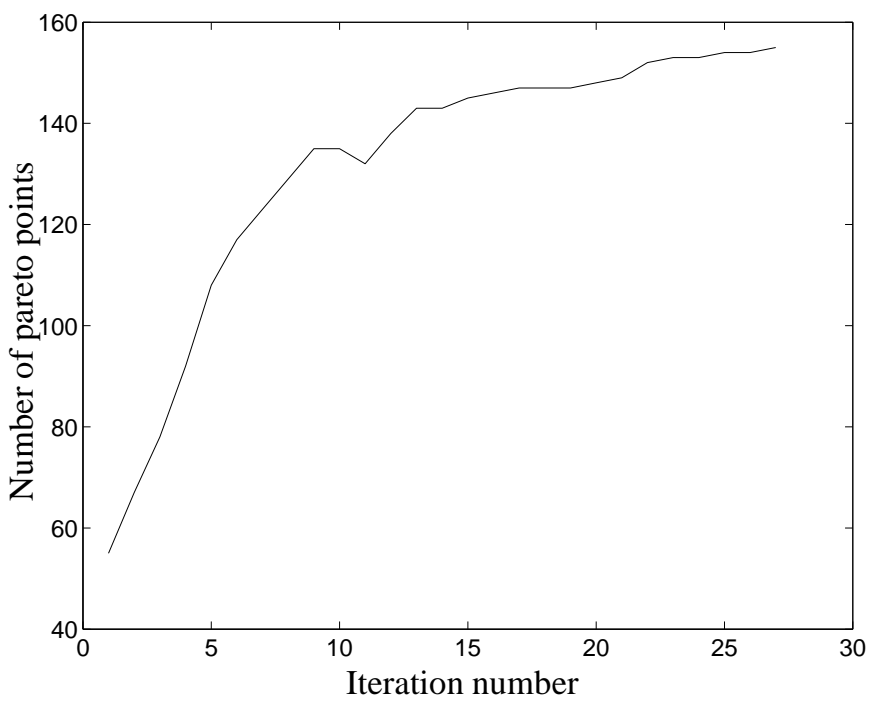

Figure 13: Evolution of the number of pareto points 


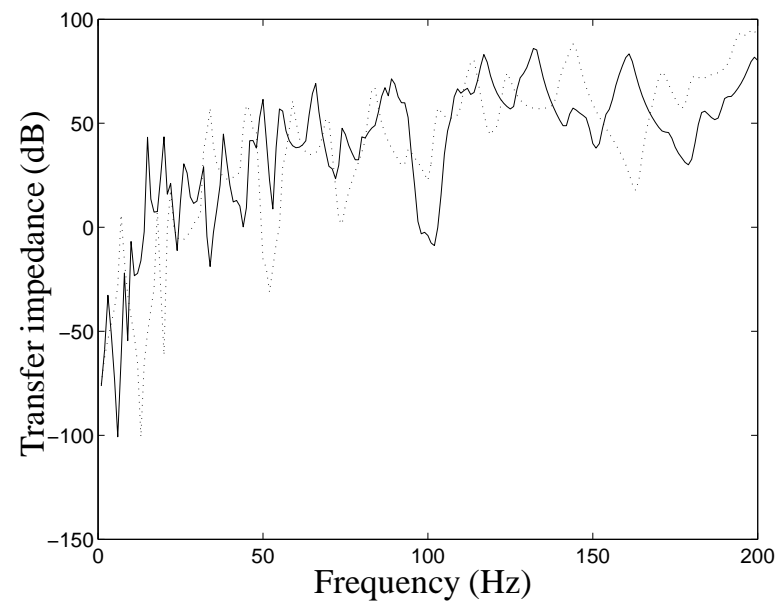

Figure 14: Square speed of a point of the plate (- : Not optimized, $\cdots$ : Optimized) 


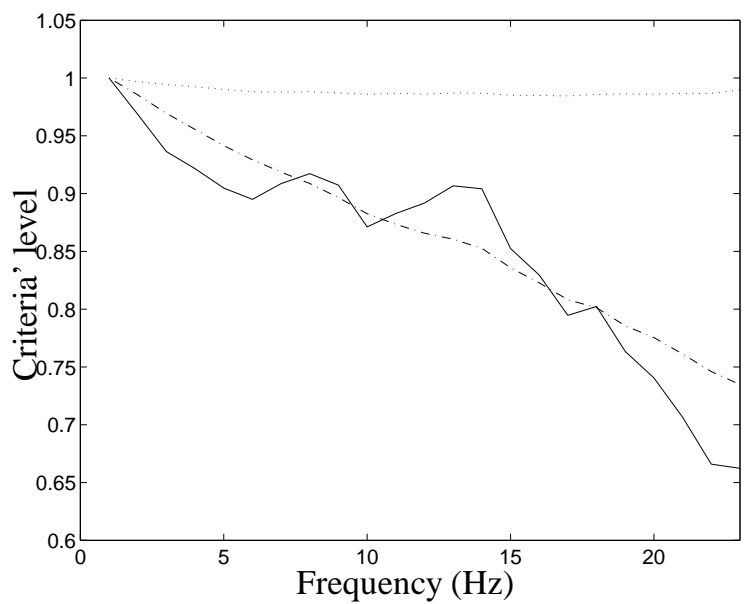

Figure 15: Evolution of the criteria (-: Mass, $\cdots: \tilde{C}_{G},-\cdot-: \tilde{C}_{T}$ ) 